\title{
Mesenchymal stem cells engineered to overexpress stem cell factor improve cardiac function but have malignant potential
}

Shafie S. Fazel, MD, PhD, Denis Angoulvant, MD, MSc, Jagdish Butany, MD, Richard D. Weisel, MD, and Ren-Ke Li, MD, PhD, Toronto, Ontario, Canada

Cardiac stem cell therapy has received significant attention as a potential biointervention to ameliorate post-myocardial infarction congestive heart failure. Recent prospective randomized clinical trials have provided some evidence showing potential efficacy, although the benefit obtained has been relatively small. ${ }^{1}$ Thus far, the majority of the evidence suggests that adult stem cells do not cause de novo cardiomyogenesis on a large and reproducible extent. Some have proposed that using embryonic stem cells, which clearly have cardiomyogenic potential, could enhance the beneficial impact of stem cell therapy. Indeed, this may be the case. However, undifferentiated embryonic stem cells are plagued with the potential for teratoma formation. ${ }^{2}$ To date, this concern has not been an issue for adult-derived stem cells.

$\mathrm{We}^{3}$ recently described the substantial functional benefit of transplanting genetically engineered adult-derived mesenchymal stem cells (MSCs) overproducing stem cell factor (SCF) when transplanted immediately after myocardial infarction. Pressure-volume loop analysis during inferior vena caval occlusion showed a 1.9 -fold increase $(P<$ $0.001)$ in the slope of preload-recruitable stroke work in animals that received MSCs genetically enhanced with SCF (MSC-SCF) compared with those that received only MSCs. The improvement in end-systolic elastance was similar to the improvement in preload-recruitable stroke work. Thus, independent of preload, MSC-SCF provided superior functional benefit. In addition, the left ventricular volume was 0.6-fold smaller $(P<.01)$ in the MSC-SCF group compared with the MSC-alone group. These benefits were clearly independent of neo-cardiomyogenesis and likely represent the paracrine influence of the transplanted cells on progenitor cell recruitment and enhancement of endogenous repair mechanism. ${ }^{3}$

Here we report extensive tumor formation in 4 of 20 mice treated with intramyocardial MSC-SCF. In the first mouse,

From the Division of Cardiac Surgery, Department of Surgery, and Department of Pathology, Toronto General Hospital, University Health Network, University of Toronto, Toronto, Ontario, Canada.

Received for publication Nov 3, 2007; accepted for publication Nov 30, 2007.

Address for reprints: Ren-Ke Li, MD, PhD, Room 3-702, Toronto Medical Discovery

Tower, Toronto General Hospital, 101 College St, Toronto, ON, Canada, M5G 1L7

(E-mail: renkeli@uhnres.utoronto.ca).

J Thorac Cardiovasc Surg 2008;136:1388-9

$0022-5223 / \$ 34.00$

Copyright (c) 2008 by The American Association for Thoracic Surgery doi: $10.1016 /$ j.jtcvs. 2007.11 .068

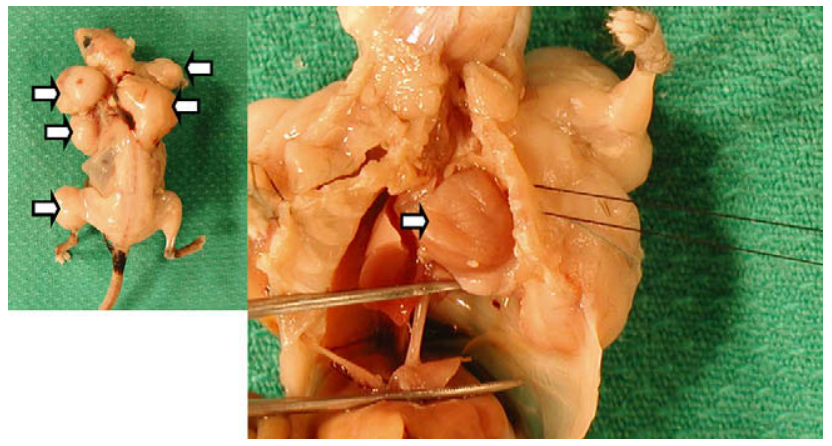

FIGURE 1. Extensive soft tissue metastatic spread of an intracardiac tumor (white arrows), which invades the chest well, is demonstrated here. This tumor was discovered 89 days after cell transplantation into the heart (arrow) in the only mouse that was not sacrificed at the end of the previous study. $^{3}$

we noted extensive metastatic disease 89 days after cell transplantation. Necropsy showed an intracardiac mass that had invaded the chest wall with gross tumor burden visible in multiple locations (Figure 1). Histologically, this tumor was a soft tissue sarcoma with greater than 10 mitotic figures per high-power field (Figure 2,A). The tumor was clearly not of thymic origin and appeared to diffusely infiltrate the myocardium (Figure 2,B). It also had both a necrotic core (Figure 2, $C$ ) and clear evidence of blood vessel invasion (Figure 2,D). All metastases were in soft tissues and no evidence of pulmonary, skeletal, or cerebral metastases could be found. The tumor stained strongly with an antibody against $\alpha$-smooth muscle actin, suggesting that it was a fibrosarcoma. Altogether, the final pathologic diagnosis was grade IV fibrosarcoma with hematogenous spread. These findings prompted more careful analysis of the histologic specimens of the 19 other animals that had already been sacrificed at day 28 for morphologic and functional evaluation. An additional 3 animals had intramyocardial fibrosarcomas detected. These mice did not have evidence for metastatic spread.

The MSCs used for these studies were in culture between 20 and 45 passages. They had stable expression of CD81 and stem cell antigen-1 but did not express c-kit (SCF receptor), CD34, CD45, or CD31. ${ }^{3}$ None of the 16 mice in the MSCalone group had tumor formation. In parallel studies looking at the impact of MSCs and MSCs engineered to overexpress tissue inhibitors of matrix metalloproteinase-3, none of the 

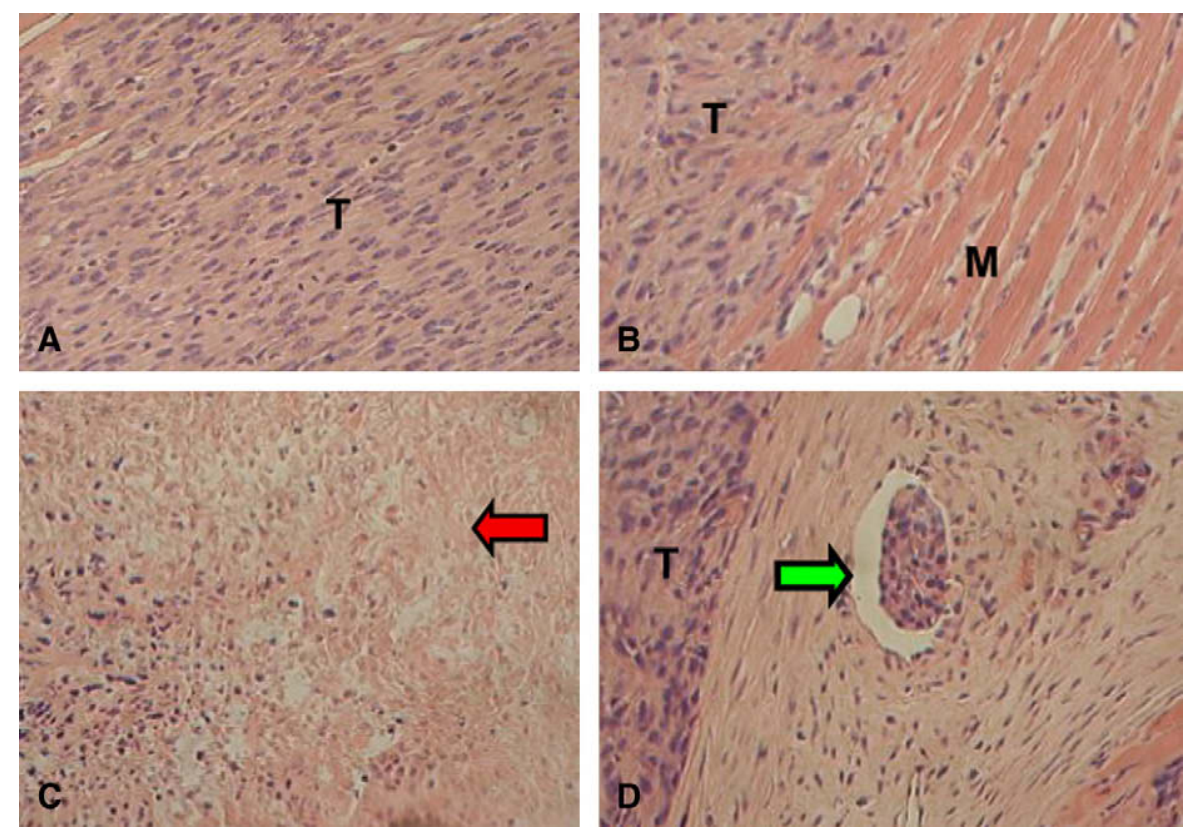

FIGURE 2. Histologic study of the tumor $(T)$ shows a very aggressive fibrosarcoma with a high mitotic index (A), with extensive intramyocardial ( $M$ ) invasion (B), a necrotic core (red arrow) attesting to rapid growth (C), and hematogenous dissemination. The green arrow points to a blood vessel (D).

total 90 mice have shown macroscopic evidence of malignancy using the same MSC line (unpublished data). The vector carrying the SCF transgene was pCDNA3 and transfection was achieved using a lipid-based technique. Stable transfectants were selected and maintained using $200 \mu \mathrm{g} /$ mL G418-spiked Iscove Modified Dulbecco's Medium over 5 additional passages. Other studies have documented the potential for MSC transformation using genetic manipulation of the Wnt pathway. ${ }^{4}$ However, MSCs up to passage 25 , have been clearly demonstrated not to develop cytogenetic abnormalities and not to undergo malignant transformation in vitro. ${ }^{5}$

Our study is the first to clearly document malignant transformation of MSCs after genetic manipulation. Although the cells had been in culture for a prolonged period, malignant transformation has not been observed in MSCalone groups or in MSCs transiently transfected with the tissue inhibitors of matrix metalloproteinase- 3 gene. Given that G418 is routinely used for selection of stable transfectant in neomycin-gene carrying plasmids without a reported case of malignant transformation to our knowledge, we are led to believe that the combination of MSCs with SCF overexpression may have increased the predisposition to malignant transformation in adult-derived MSCs. These findings raise significant concerns for attempts at gene enhancement of adult-derived stem cells for myocardial repair. This conclusion comes with a critical caveat that the malignant transformation was caused by the specific combination of techniques that we used to generate our cell line.

\section{References}

1. Rosenzweig A. Cardiac cell therapy-mixed results from mixed cells. $N$ Engl J Med. 2006;355:1274-7.

2. Cao F, Van Der Bogt KE, Sadrzadeh A, Xie X, Sheikh AY, Wang H, et al. Spatial and temporal kinetics of teratoma formation from murine embryonic stem cell transplantation. Stem Cells Dev. 2007;16:883-91.

3. Fazel S, Chen L, Weisel RD, Angoulvant D, Seneviratne C, Fazel A, et al. Cell transplantation preserves cardiac function after infarction by infarct stabilization: augmentation by stem cell factor. J Thorac Cardiovasc Surg. 2005;130:1310.

4. Matushansky I, Hernando E, Socci ND, Mills JE, Matos TA, Edgar MA, et al. Derivation of sarcomas from mesenchymal stem cells via inactivation of the Wnt pathway. J Clin Invest. 2007;117:3248-57.

5. Bernardo ME, Zaffaroni N, Novara F, Cometa AM, Avanzini MA, Moretta A, et al. Human bone marrow derived mesenchymal stem cells do not undergo transformation after long-term in vitro culture and do not exhibit telomere maintenance mechanisms. Cancer Res. 2007;67:9142-9. 